\title{
Mycobacterial signaling through toll-like receptors
}

\author{
Joyoti Basu' ${ }^{1}$ Dong-Min Shin ${ }^{2}$ and Eun-Kyeong $\mathrm{Jo}^{2 *}$ \\ 1 Department of Chemistry, Bose Institute, Kolkata, India \\ ${ }^{2}$ Department of Microbiology and Infection Signaling Network Research Center, Chungnam National University School of Medicine, Daejeon, South Korea
}

Edited by:

Nelson Gekara, Umea University,

Sweden

Reviewed by:

Maximiliano G. Gutierrez, Medical

Research Council- National Institute for Medical Research, UK

Javeed Ali Shah, University of

Washington, USA

*Correspondence:

Eun-Kyeong Jo, Department of

Microbiology and Infection Signaling

Network Research Center,

Chungnam National University

School of Medicine, 301-747,

Daejeon, South Korea.

e-mail: hayoungj@cnu.ac.kr
Studies over the past decade have helped to decipher molecular networks dependent on Toll-like receptor (TLR) signaling, in mycobacteria-infected macrophages. Stimulation of TLRs by mycobacteria and their antigenic components rapidly induces intracellular signaling cascades involved in the activation of nuclear factor-kB and mitogen-activated protein kinase pathways, which play important roles in orchestrating proinflammatory responses and innate defense through generation of a variety of antimicrobial effector molecules. Recent studies have provided evidence that mycobacterial TLR-signaling cross talks with other intracellular antimicrobial innate pathways, the autophagy process and functional vitamin $D$ receptor (VDR) signaling. In this article we describe recent advances in the recognition, responses, and regulation of mycobacterial signaling through TLRs.

Keywords: mycobacteria, vitamin D, autophagy, antimicrobial peptides, innate immunity

\section{INTRODUCTION}

Tuberculosis remains a serious health problem worldwide, causing an alarming two million deaths annually (WHO, 2010). One-third of the global population is latently infected with Mycobacterium tuberculosis (Mtb). In this state, healthy immunecompetent individuals are able to combat infection by mounting of an effective immune response (Huynh et al., 2011). When the immune system is compromised, the bacteria are capable of replicating, disseminating, and causing a progressive infectious disease (Huynh et al., 2011). Thus, appropriate and efficient mounting of host immune responses are required for controlling tuberculosis infection. Protective immunity to mycobacteria mainly requires an interplay between innate immune responses and Th1-dependent cellular immune responses, which cooperatively contribute to clear the major human pathogen, Mtb (Zuñiga et al., 2012).

During infection of macrophages, Mtb and its components encounters innate immunity, which operates through a variety of germline-encoded pattern recognition receptors including Toll-like receptors (TLRs) for recognition of various molecular patterns of mycobacteria (Jo et al., 2007; Natarajan et al., 2011). The innate immune response triggered by engagement of TLRs, involves recruitment of cytoplasmic adaptor proteins and intracellular signaling molecules resulting in phagosome maturation and the synthesis of pro-inflammatory cytokines (Sundaramurthy and Pieters, 2007; Natarajan et al., 2011). Antimicrobial responses through TLR-dependent triggering of intracellular signaling cascades, involves the activation of nuclear factor (NF)- $\mathrm{kB}$ in murine macrophages and vitamin $\mathrm{D}$ receptor (VDR) signaling in human monocytes/macrophages (Sundaramurthy and Pieters, 2007; Jo, 2010; Yuk et al., 2012). Recent studies have also highlighted that the autophagy pathway is critically involved in degradation/elimination of intracellular microbes, including Mtb (Deretic, 2012; Mintern and Villadangos, 2012). Pathogenic mycobacteria utilize a unique strategy to survive within macrophages. They have developed tactics to escape from host autophagic process. They also expose multiple ligands that are recognized by host TLRs. An understanding of the complex mechanisms involved in this hide-and-seek game (host-pathogen interaction) is required for development of novel intervention strategies to combat tuberculosis.

In this Review, we focus on emerging data showing that mycobacteria and their ligands activate or modulate TLR signaling pathways. Specifically, we discuss the mechanisms by which TLR signaling regulates the initiation and progression of innate immune responses, and eradication of intracellular mycobacteria through cooperation with autophagy and VDR signaling pathway.

\section{OVERVIEW OF TLRS IN MYCOBACTERIAL INFECTION}

Mtb primarily invades the lung alveoli via inhalation and alveolar macrophages are the principal cell types infected by this pathogen (Huynh et al., 2011). When Mtb are internalized by alveolar macrophages or other innate cells, they encounter a variety of intracellular innate receptors including TLRs. So far, there is evidence that mycobacterial components are engaged by TLR2 in association with TLR1/TLR6, by TLR4, or by TLR9 (which recognizes Mtb DNA) (Doherty and Arditi, 2004; Jo et al., 2007). Earlier in vivo studies showed somewhat discordant results in susceptibility of mice deficient in several TLR-related genes, including TLR2, TLR4, TLR6, or MyD88, in Mtb infection (Doherty and Arditi, 2004; Quesniaux et al., 2004). It was also reported that TLR2/4/9-deficient mice have no apparent difference in activation of antigen-specific $\mathrm{T}$ cells, and production of pro-inflammatory cytokines and interferon (IFN) $-\gamma$, when compared with wild-type mice (Hölscher et al., 2008). The controversial results might be 
due to differences in Mtb strains used, doses of infection, differences in genetic backgrounds, etc. The detailed description about the in vivo role of TLRs in mycobacterial infection is summarized in excellent earlier reviews by Stenger and Modiln (2002); Doherty and Arditi (2004); and Quesniaux et al. (2004). However, the exact roles and regulatory mechanisms by which TLRs affect innate immunity and antimicrobial responses have not been fully characterized.

Numerous studies have revealed that TLR2 is involved in the innate recognition and responses in innate immune cells such as macrophages and dendritic cells of a variety of mycobacterial cell wall antigens including $19-\mathrm{kDa}$ mycobacterial lipoprotein, glycolipids like lipoarabinomannan (LAM), LM, 38-kDa antigen, LprG lipoprotein, phosphatidylinositol mannoside (PIM), triacylated (TLR2/TLR1), or diacylated (TLR2/TLR6) lipoproteins [reviewed in Jo et al. (2007); Kleinnijenhuis et al. (2011)]. TLR4 is activated by heat shock protein $60 / 65$ and $38-\mathrm{kDa}$ antigen, whereas TLR9 recognizes unmethylated CpG motifs of mycobacterial DNA [reviewed by Jo et al. (2007); Kleinnijenhuis et al. (2011)]. Stimulation of TLRs by mycobacterial ligands leads to an initiation of intracellular signaling cascades culminating in proinflammatory cytokine generation in macrophages and dendritic cells through activation of NF- $\mathrm{B}$ and MAPK pathways (Jo et al., 2007, summarized in Figure 1). Along with canonical NF- $\kappa$ B and
MAPK-dependent signaling, it may also be noted that the transcription factor nuclear factor of activated T cells 5 (NFAT5) can be activated by TLR signaling induced by Mtb stimulation, and the co-infection of HIV-1 in tuberculosis accelerates an increase of viral load through expression of NFAT5 (Ranjbar et al., 2012). Recent reports have highlighted that other cells such as alveolar epithelial cells play an important role in innate defense to produce the chemokine CCL2 and also in the pathogenesis of mycobacterial infection (Chuquimia et al., 2012). Additionally, mast cells are activated by mycobacterial LAM to induce the generation of cysteinyl leukotriene through TLR2-NF- $\kappa \mathrm{B}$-dependent signaling (Bąbolewska et al., 2012). Together, these studies suggest that TLRs have a crucial role in the host immune responses against mycobacterial infection and that they are involved in not only immune cells but also other cells. These findings have been summarized in Figure 1. In this context, it is pertinent to note that while, to the best of our knowledge, there is no detailed report on the temporal dynamics of NF- $\kappa \mathrm{B}$ activation during infection, our (Basu laboratory) unpublished observations from ex vivo experiments using Mtb-infected macrophages, indicate that the upregulation of NF- $\kappa \mathrm{B}$ dampening molecules such as TNFAIP3 (A20) occurs as early as $24 \mathrm{~h}$ post-infection. It is obvious that Mtb elicits inflammation-dampening signals in macrophages which would favor its survival.

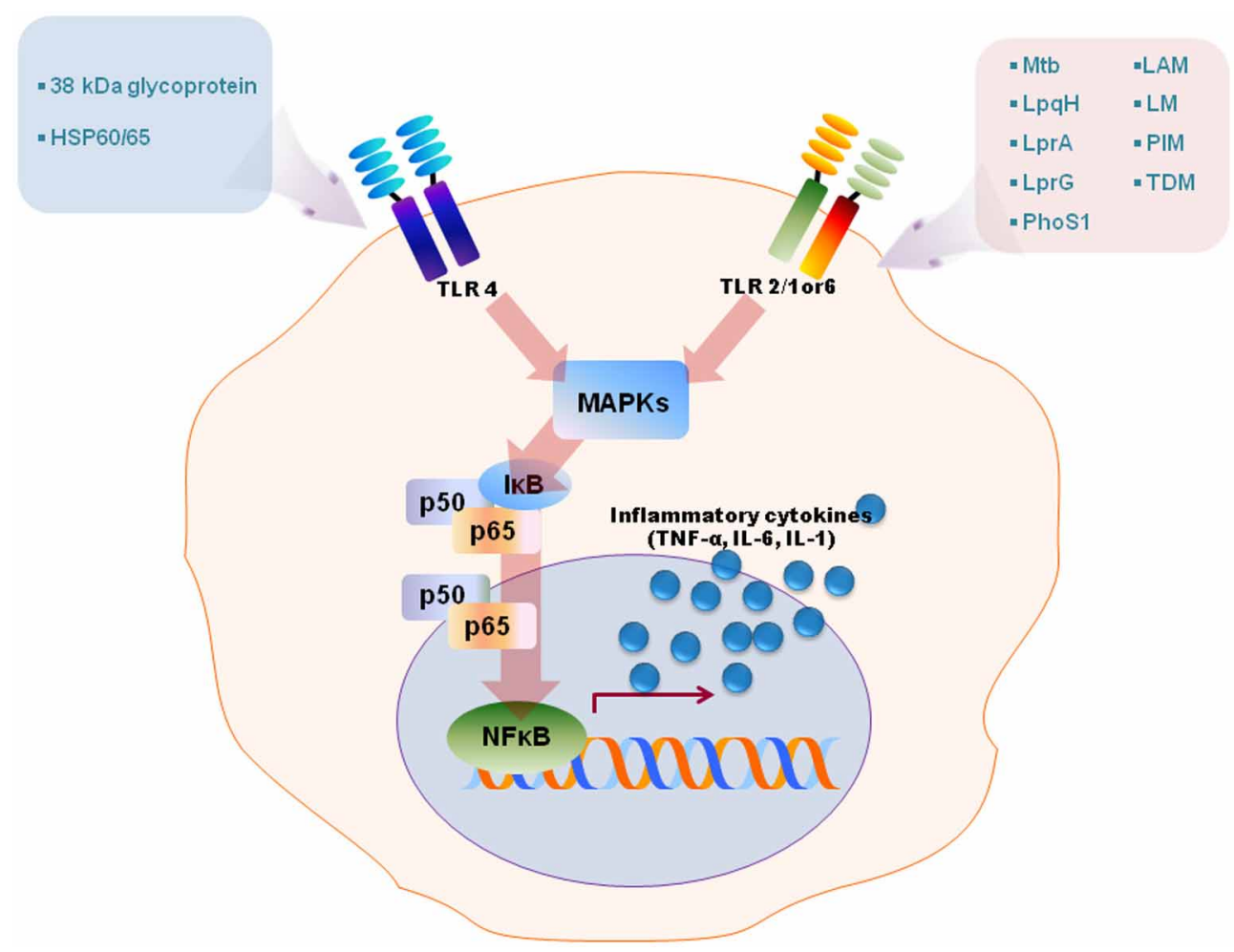

FIGURE 1 | A schematic model for TLRs activation by diverse mycobacterial antigens. TLRs are involved in the innate recognition and responses in innate immune cells to numerous mycobacterial antigens. Some mycobacterial antigens including LpqH, lipoarabinomannan (LAM), lipomannan (LM), 38-kDa antigen, LprG, LprA, PhoS1, trehalose dimycolate
(TDM), phosphatidylinositol mannoside (PIM) activate TLR2/1 or 6, whereas TLR4 recognizes heat shock protein (HSP) 60/65 and 38-kDa antigen. TLR activation by mycobacterial antigens leads an intracellular signaling pathway that culminates in the production of proinflammatory in macrophages and dendritic cells through MAPK and NF-kB pathways. 


\section{TLRS SIGNALING BY MYCOBACTERIAL COMPONENTS}

Many previous studies have reported that mycobacterial components are involved in innate recognition and responses through TLR signaling (Basu et al., 2007; Pathak et al., 2009; Bansal et al., 2010, 2011; Heo et al., 2011; Byun et al., 2012a,b). Antigens of the PE_PGRS family, namely, PE_PGRS 17 (Rv0978c) and PE_PGRS 11 (Rv0754), recognize TLR2 to induce maturation and activation of human dendritic cells, and enhance the ability of dendritic cells to stimulate CD4(+) T cells (Bansal et al., 2010). These PE_PGRS proteins activate dendritic cells through ERK1/2 and p38 MAPK signaling pathways. Basu et al. have shown that PE_PGRS 33 signals through TLR2 to release TNF- $\alpha$ to induce apoptosis of macrophages (Basu et al., 2007).

Other Mtb proteins have been shown to influence dendritic cell function. Rv0462 (lipoamide dehydrogenase C), Rv0315, and Rv0577 (Mtb-restricted secretory protein involved in the methylglyoxal detoxification pathway) induce dendritic cell maturation and activation leading to increased expression of costimulatory molecules (CD80, CD86, and class II MHC) and proinflammatory cytokines (TNF- $\alpha$, IL-1 $\beta$, IL-6, and IL-12), and potentiate the Th1 immune response (Heo et al., 2011; Byun et al., 2012a,b). These findings have been summarized in Figure 2 .
Yet another effecter of proinflammatory cytokine production is trehalose dimycolate (TDM). TDM has been reported to be tethered to several receptors, including TLR2, the class A scavenger receptor MARCO, Fc receptor- $\gamma(\mathrm{FcR} \gamma)$, and macrophage-inducible C-type lectin (Mincle) (Bowdish et al., 2009; Ishikawa et al., 2009; Werninghaus et al., 2009). TDM triggers MARCO/TLR2/CD14-dependent signaling to produce proinflammatory cytokines (Bowdish et al., 2009). In addition to TLR2-dependent signaling, TDMs also activate macrophages and dendritic cells via FcR $\gamma$-Syk-Card9 pathway (Werninghaus et al., 2009). Mincle, which is one of the C-type lectin receptors expressed in macrophages subjected to several types of stress (Matsumoto et al., 1999), is an essential receptor for TDMdependent inflammatory responses, nitric oxide synthesis, and granuloma formation (Ishikawa et al., 2009). TDM receptors are associated with partial protection against Mtb infection (Bowdish et al., 2009; Ishikawa et al., 2009; Werninghaus et al., 2009).

Recent work by Bansal et al. (2011) suggests that upon infection with $M$. bovis bacille Calmette-Guérin (BCG), TLR2 signaling activates Wnt- $\beta$-catenin signaling. The authors suggest that TLR2 integrates Wnt- $\beta$-catenin signaling to modulate a battery of genes associated with $\mathrm{T}(\mathrm{Reg})$ cell lineage commitment. At low MOIs, Mtb-triggered macrophage apoptosis depends on a TLR2 signaling cascade associated with ASK1/p38 MAPK- and

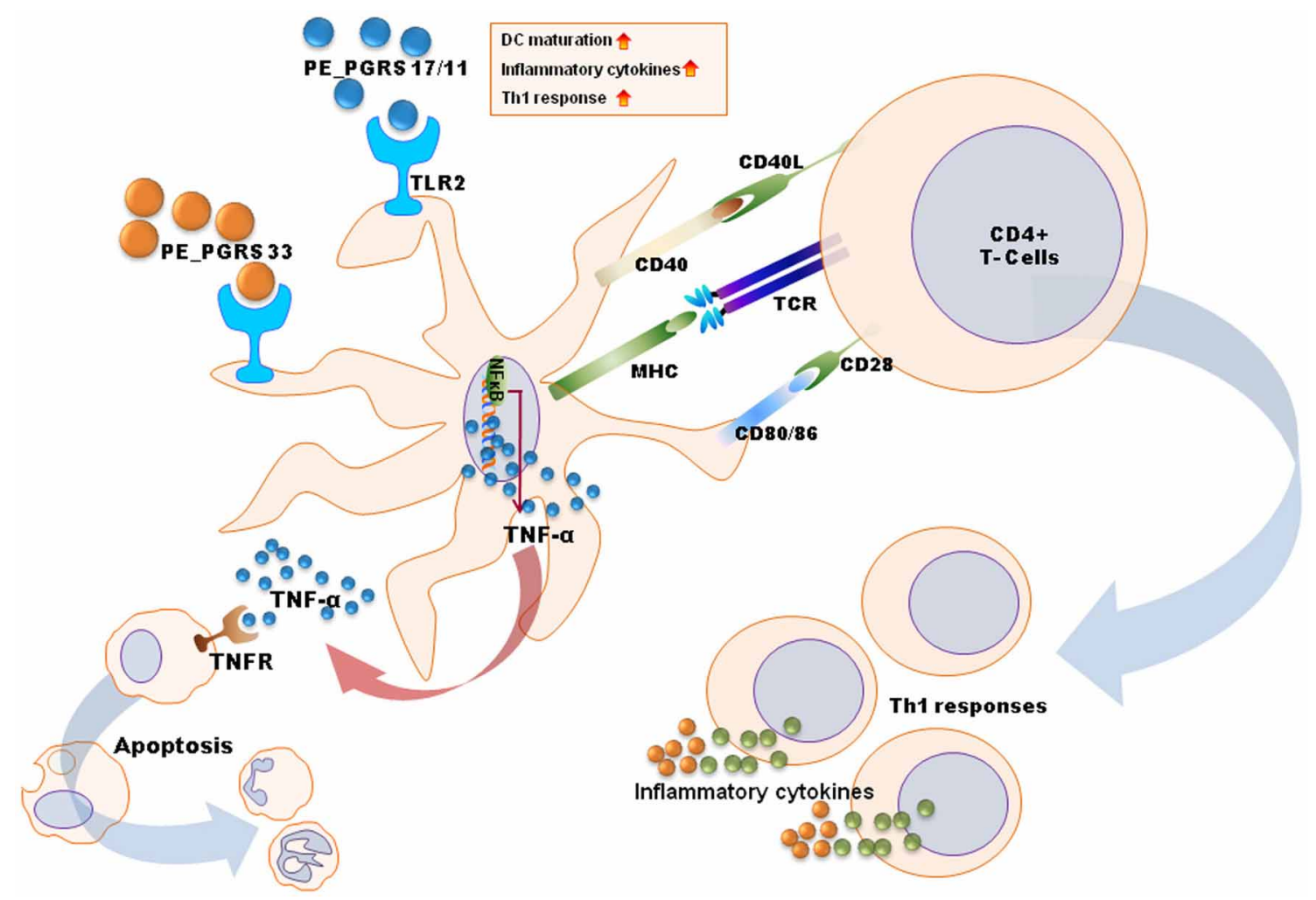

FIGURE 2 | A schematic diagram for mechanisms of dendritic cell maturation/activation to stimulate $T$ cells by mycobacterial antigens. PE_PGRS proteins activate dendritic cells through TLR2-MAPK-NF-KB signaling pathways. PE_PGRS 33-induced TNF- $\alpha$ by dendritic cells induces apoptosis in macrophages. Others (e.g., Rv0462, Rv0315, Rv0577, and PE_PGRS 17/11) increase expression of costimulatory molecules (CD80, CD86, and class II MHC) and proinflammatory cytokines (TNF- $\alpha$, IL-1 $\beta$, IL-6, and IL-12), leading to Th1 immune responses. 
c-Abl-dependent phosphorylation of c-FLIP and its degradation, leading to caspase 8 activation (Kundu et al., 2009).

TLR-dependent NF- $\kappa \mathrm{B}$ signaling and MAPK pathways contribute to antimycobacterial innate immunity through secretion of antibacterial effector molecules, cytokines, and chemokines, thus recruiting various immune cells to the site of infection (Jo et al., 2007; Jo, 2010; Huynh et al., 2011; Kleinnijenhuis et al., 2011). In contrast, Mtb-mediated TLR2 signaling is associated with generation of an environment favoring the survival of Mtb in macrophages (Yoshida et al., 2009). Together, TLR signaling in mycobacterial infection acts as a double-edged sword regulating a delicate balance in host innate and inflammatory responses to determine disease outcome.

\section{HUMAN TLR POLYMORPHISM AND MYCOBACTERIAL INFECTION}

Human genetic polymorphisms of TLRs have a role in regulating innate recognition of microbes and in determining susceptibility to mycobacterial infection. The relationship between TLR polymorphisms, disease susceptibility, and innate immune activities against mycobacteria has been described in several reviews (Texereau et al., 2005; Lykouras et al., 2008; Kleinnijenhuis et al., 2011). Here we describe the recent data on genetic and functional studies of TLRs in protection and pathogenesis of mycobacterial infection. It has been reported that TLR1/6-deficient genotypes (typified by TLR1_T1805G and TLR6_C745T), after vaccination with $M$. bovis BCG, are linked with the increased production of Th1-type $\mathrm{T}$ cell cytokines through regulation of monocyte IL-10 production (Randhawa et al., 2011). Other recent studies have reported that $\mathrm{rs} 352139$, an SNP located in the intron of TLR9 is associated with tuberculosis susceptibility in Indonesian and Vietnamese populations (Kobayashi et al., 2012). It has also been reported that TLR8 polymorphisms are associated with pulmonary tuberculosis susceptibility in males (Davila et al., 2008). Interestingly, TLR8 transcriptional levels are increased in patients at the early phase of infection and TLR8 protein expression is increased in macrophages after M. bovis BCG infection (Davila et al., 2008).

Previously, it was shown that human TLR1 deficiency is linked with impaired mycobacterial innate immune signaling and susceptibility to leprosy (Misch et al., 2008). Recent studies have shown that TLR chaperones, PRAT4A, and PRAT4B are important regulators in TLR1 trafficking to the cell surface and are also regulated by IFN- $\gamma$ (Hart and Tappiung, 2012). Furthermore, there is an inverse correlation between Euro-American lineage of $\mathrm{Mtb}$ and extra-pulmonary dissemination, suggesting that a link between interaction between host and mycobacterial genotypes and the clinical progression of tuberculosis (Caws et al., 2008). Future studies are warranted to define the exact relationship between host and bacterial genotype and disease phenotype using clinical isolates.

\section{CROSS TALK BETWEEN TLR, AUTOPHAGY, AND VITAMIN D SIGNALING PATHWAYS}

Several other innate immune pathways are closely linked with the TLR signaling pathway, thus cooperatively defining the innate immune response which enables clearance of mycobacteria inside cells. Activation of autophagy has a critical effector role in innate immune responses such as enhancement of phagosomal maturation and coordination of the innate and adaptive immune systems (Deretic, 2009, 2012). It is known that a variety of pathogenor danger-associated molecular patterns and pattern-recognition receptor signaling are closely associated with autophagy activation (Xu et al., 2007; Delgado et al., 2008; Fabri et al., 2011a; Deretic, 2012). Therefore, it is not surprising that TLR signaling pathways triggered by engagement of mycobacterial antigens lead to activation of autophagy (Shin et al., 2010). The activation of autophagy influences the antigen-presenting capacity of the immunodominant mycobacterial antigen Ag85B by antigenpresenting cells (Jagannath et al., 2009), as well as the production of antimicrobial proteins and pro-inflammatory cytokines (Yuk et al., 2009; Shin et al., 2010), thereby influencing vaccine efficacy and host immune defense during mycobacterial infection.

Recent studies have uncovered the interplay of vitamin D-dependent antimicrobial responses and autophagy pathways in the activation of host defense against mycobacterial infection (Yuk et al., 2009; Jo, 2010; Shin et al., 2010; Fabri et al., 2011a). Activation of TLR signaling in human monocytes/macrophages has been shown to lead to the induction of the antimicrobial peptide cathelicidin, which is critically involved in antimicrobial responses against Mtb (Liu et al., 2006, 2007; Yang et al., 2009). Physiological concentrations of $1,25 \mathrm{D} 3$ were found to be enough to induce the production of the human cathelicidin and autophagic flux, thereby activating antimicrobial activities against $\mathrm{Mtb}$ and also inhibiting HIV replication in cells co-infected with Mtb and HIV (Yuk et al., 2009; Campbell and Spector, 2012). Moreover, cathelicidin plays a dual role in vitamin D-induced autophagy activation in human monocytes/macrophages, acting both as a critical effector of antimicrobial responses against $\mathrm{Mtb}$ and as a mediator of the autophagy pathway through enhancement of autophagic flux and the induction of autophagy-related genes (Yuk et al., 2009; Campbell and Spector, 2012). Importantly, in human macrophages cultured with vitamin D-sufficient sera, IFN- $\gamma$ production by Th1 cells as well as stimulation of TLR2/1, turn on antimicrobial activity through antimicrobial peptide expression, autophagy activation, and phagosome-lysosome fusion (Fabri et al., 2011b). These findings provide insights into how vitamin D-dependent signaling triggers antimicrobial activity by activating autophagy and regulating both innate and adaptive immune responses.

The impact of host autophagy on phagosome maturation and Mtb killing gives rise to the idea that compounds that activate or modulate the autophagy pathway, could potentially enhance antimicrobial activities against Mtb infection (Gutierrez et al., 2004; Singh et al., 2006; Fabri et al., 2011a; Kim et al., 2012; Lam et al., 2012; Zullo and Lee, 2012). Recent studies have shown that the prevalent anti-TB drugs isoniazid and pyrazinamide promote autophagy activation. This plays an important role in successful antimicrobial responses during therapy against mycobacterial infection in vivo and in vitro (Kim et al., 2012). Lam et al. also showed that the antiprotozoal drug nitazoxanide stimulates autophagy induction and mTORC1 inhibition, and inhibits Mtb 
proliferation in human monocytes and monocytic cells (Lam et al., 2012).

\section{CONCLUDING REMARKS}

Developing vaccines and chemotherapeutic agents is the cornerstone of the successful management of tuberculosis. Understanding the molecular mechanisms of specific interactions of Mtb with its host should augment efforts in both these areas. Immune modulation as a strategy to combat mycobacterial infection remains underexplored. This review brings to light how mycobacterial modulins signaling through TLRs, contribute either to an effective host immune response or to immune evasion by the pathogen. Targeting those modulins that facilitate immune evasion, or exploiting those that facilitate a robust innate and adaptive immune response, could offer new avenues for controlling infection. The review also brings forth the

\section{REFERENCES}

Bạbolewska, E., Witczak, P., Pietrzak, A., and Brzeziñska-Błaszczyk, E. (2012). Different potency of bacterial antigens TLR2 and TLR4 ligands in stimulating mature mast cells to cysteinyl leukotriene synthesis. Microbiol. Immunol. 56, 183-190.

Bansal, K., Elluru, S. R., Narayana, Y., Chaturvedi, R., Patil, S. A., Kaveri, S. V., et al. (2010). PE_PGRS antigens of Mycobacterium tuberculosis induce maturation and activation of human dendritic cells. J. Immunol. 184, 3495-3504.

Bansal, K., Trinath, J., Chakravortty, D., Patil, S. A., and Balaji, K. N. (2011). Pathogen-specific TLR2 protein activation programs macrophages to induce Wnt-betacatenin signaling. J. Biol. Chem. 286, 37032-37044.

Basu, S., Pathak, S. K., Banerjee, A., Pathak, S., Bhattacharyya, A., Yang, Z., et al. (2007). Execution of macrophage apoptosis by PE_PGRS33 of Mycobacterium tuberculosis is mediated by Toll-like receptor 2-dependent release of tumor necrosis factor- $\alpha$. J. Biol. Chem. 282, 1039-1050.

Bowdish, D. M. E., Sakamoto, K., Kim, M.-J., Kroos, M., Mukhopadhyay, S., Leifer, C. A., et al. (2009). MARCO, TLR2, and CD14 are required for macrophage cytokine responses to mycobacterial trehalose dimycolate and Mycobacterium tuberculosis. PLoS Pathog. 5:e1000474. doi: 10.1371/journal.ppat.1000474

Byun, E. H., Kim, W. S., Kim, J. S., Jung, I. D., Park, Y. M., Kim, H. J., et al. (2012a). Mycobacterium tuberculosis Rv0577, a novel TLR2 agonist, induces maturation of dendritic cells and drives Th1 2695-2711.

Byun, E. H., Kim, W. S., Shin, A. R., Kim, J. S., Whang, J., Won, C. J., et al. (2012b). Rv0315, a novel immunostimulatory antigen of Mycobacterium tuberculosis activates dendritic cells and drives Th1 immune responses. J. Mol. Med. (Berl.) 90, 285-298.

Campbell, G. R., and Spector, S. A. (2012). Vitamin D inhibits human immunodeficiency virus type 1 and Mycobacterium tuberculosis infection in macrophages through the induction of autophagy. PLoS Pathog. 8:e1002689. doi: 10.1371/journal.ppat.1002689

Caws, M., Thwaites, G., Dunstan, S., Hawn, T. R., Lan, N. T., Thuong, N. T., et al. (2008). The influence of host and bacterial genotype on the development of disseminated disease with Mycobacterium tuberculosis. PLoS Pathog. 4:e1000034. doi: 10.1371/journal.ppat.1000034

Chuquimia, O. D., Petursdottir, D. H., Rahman, M. J., Hartl, K., Singh, M., and Fernández, C. (2012). The role of alveolar epithelial cells in initiating and shaping pulmonary immune responses: communication between innate and adaptive immune systems. PLoS ONE 7:e32125. doi: 10.1371/journal.pone.0032125

Davila, S., Hibbeerd, M. L., Hari Dass, R., Wong, H. E., Sahiratmadja, E., Bonnard, C., et al. (2008). Genetic association and expression studies indicate a role of toll-like receptor 8 in pulmonary tuberculosis. PLoS Genet. 4:e1000218. doi: 10.1371/journal.pgen.1000218

Delgado, M. A., Elmaoued, R. A., Davis, A. S., Kyei, G., and Deretic, V. (2008). Toll-like receptors immune response. FASEB J. 26,

attractive possibility of developing therapeutics designed to augment autophagy as a means of restricting mycobacterial survival and combating infection.

\section{ACKNOWLEDGMENTS}

We are indebted to current and past members of our laboratory for discussions and investigations that contributed to this article. This work was supported by the Korea Science \& Engineering Foundation through the Infection Signaling Network Research Center (R13-2007-020-01000-0) at Chungnam National University (Eun-Kyeong Jo), and by a grant (INT/Korea/P-10) from the Department of Science and Technology, Government of India (Joyoti Basu) under the IndoKorea Joint Program of Cooperation in Science and Technology. We apologize to colleagues whose work and publications could not be referenced owing to space constraints.

control autophagy. EMBO J. 27, $1110-1121$.

Deretic, V. (2009). Multiple regulatory and effector roles of autophagy in immunity. Curr. Opin. Immunol. 21, 53-62.

Deretic, V. (2012). Autophagy as an innate immunity paradigm: expanding the scope and repertoire of pattern recognition receptors. Curr. Opin. Immunol. 24, 21-31.

Doherty, T. M., and Arditi, M. (2004). $\mathrm{TB}$, or not TB: that is the question does TLR signaling hold the answer? J. Clin. Invest. 114, 1699-1703.

Fabri, M., Realegeno, S. E., Jo, E. K., and Modlin, R. L. (2011a). Role of autophagy in the host response to microbial infection and potential for therapy. Curr. Opin. Immunol. 23, 65-70.

Fabri, M., Stenger, S., Shin, D. M., Yuk, J. M., Liu, P. T., Realegeno, S., et al. (2011b). Vitamin D is required for IFN-gamma-mediated antimicrobial activity of human macrophages. Sci. Transl. Med. 3, 104 ra102.

Gutierrez, M. G., Master, S. S., Singh, S. B., Taylor, G. A., Colombo, M. I., and Deretic, V. (2004). Autophagy is a defense mechanism inhibiting BCG and Mycobacterium tuberculosis survival in infected macrophages. Cell 119, 753-766.

Hart, B. E., and Tappiung, R. I. (2012). Cell surface trafficking of TLR1 is differentially regulated by the chaperones PRAT4A and PRAT4B. J. Biol. Chem. 287, 16550-16562.

Heo, D. R., Shin, S. J., Kim, W. S., Noh, K. T., Park, J. W., Son, K. H., et al. (2011). Mycobacterium tuberculosis lpdC, Rv0462, induces dendritic cell maturation and Th1 polarization. Biochem. Biophys. Res. Commun. 411, 642-647.
Hölscher, C., Reiling, N., Schaible, U. E., Hölscher, A., Bathmann, C., Korbel, D., et al. (2008). Containment of aerogenic Mycobacterium tuberculosis infection in mice does not require MyD88 adaptor function for TLR2, -4 and -9. Eur. J. Immunol. 38, 680-694.

Huynh, K. K., Joshi, S. A., and Brown, E. J. (2011). A delicate dance: host response to mycobacteria. Curr. Opin. Immunol. 23, 464-472.

Ishikawa, E., Ishikawa, T., Morita, Y. S., Toyonaga, K., Yamada, H., Takeuchi, O., et al. (2009). Direct recognition of the mycobacterial glycolipid, trehalose dimycolate, by C-type lectin Mincle. J. Exp. Med. 206, 2879-2888.

Jagannath, C., Lindsey, D. R., Dhandayuthapani, S., Xu, Y., Hunter, R. L. Jr., and Eissa, N. T. (2009). Autophagy enhances the efficacy of $\mathrm{BCG}$ vaccine by increasing peptide presentation in mouse dendritic cells. Nat. Med. 15, 267-276.

Jo, E. (2010). Innate immunity to mycobacteria: vitamin D and autophagy. Cell. Microbiol. 12, 1026-1035.

Jo, E. K., Yang, C. H., Choi, C. H., and Harding, C. V. (2007). Intracellular signalling cascades regulating innate immune responses to Mycobacteria: branching out from Toll-like receptors. Cell. Microbiol. 9, 1087-1098.

Kim, J. J., Lee, H. M., Shin, D. M., Kim, W., Yuk, J. M., Jin, H. S., et al. (2012). Host cell autophagy activated by antibiotics is required for their effective antimycobacterial drug action. Cell Host Microbe 11, 457-468.

Kleinnijenhuis, J., Oosting, M., Joosten, L. A., Netea, M. G., and Van Crevel, 
R. (2011). Innate immune recognition of Mycobacterium tuberculosis. Clin. Dev. Immunol. 2011, 405310.

Kobayashi, K., Yuliwulandari, R., Yanai, H., Naka, I., Lien, L. T., Hang, N. T., et al. (2012). Association of TLR polymorphisms with development of tuberculosis in Indonesian females. Tissue Antigens 79, 190-197.

Kundu, M., Pathak, S. K., Kumawat, K., Basu, S., Chatterjee, G., Pathak, S., et al. (2009). A TNF-and c-Cbldependent FLIP(S)-degradation pathway and its function in Mycobacterium tuberculosisinduced macrophage apoptosis. Nat. Immunol. 10, 918-926.

Lam, K. K., Zheng, X., Forestieri, R., Balgi, A. D., Nodwell, M., Vollett, S., et al. (2012). Nitazoxanide stimulates autophagy and inhibits mTORC1 signaling and intracellular proliferation of Mycobacterium tuberculosis. PLoS Pathog. 8:e1002691. doi: 10.1371/journal.ppat.1002691

Liu, P. T., Stenger, S., Li, H., Wenzel, L., Tan, B. H., Krutzik, S. R., et al. (2006). Toll-like receptor triggering of a vitamin D-mediated human antimicrobial response. Science 311, 1770-1773.

Liu, P. T., Stenger, S., Tang, D. H., and Modlin, R. L. (2007). Cutting edge: vitamin $\mathrm{D}$-mediated human antimicrobial activity against Mycobacterium tuberculosis is dependent on the induction of cathelicidin. J. Immunol. 179, 2060-2063.

Lykouras, D., Sampsonas, F., Kaparianos, A., Karkoulias, K., Tsoukalas, G., and Spiropoulos, K. (2008). Human genes in TB infection: their role in immune response. Monaldi Arch. Chest Dis. 69, 24-31.

Matsumoto, M., Tanaka, T., Kaisho, T., Sanjo, H., Copeland, N. G., Gilbert, D. J., et al. (1999). A novel LPS-inducible C-type lectin is a transcriptional target of NF-IL6 in macrophages. J. Immunol. 163, 5039-5048.

Mintern, J. D., and Villadangos, J. A. (2012). Autophagy and mechanisms of effective immunity. Front. Immunol. 3:60. doi: 10.3389/fimmu.2012.00060

Misch, E. A., Macdonald, M., Ranjit, C., Sapkota, B. R., Wells, R. D., Siddiqui, M. R., et al. (2008). Human TLR1 deficiency is associated with impaired mycobacterial signaling and protection from leprosy reversal reaction. PLoS Negl. Trop. Dis. 2:e231. doi: 10.1371/journal.pntd.0000231

Natarajan, K., Kundu, M., Sharma, P., and Basu, J. (2011). Innate immune responses to $M$. tuberculosis infection. Tuberculosis (Edinb.) 91, 427-431.

Pathak, S., De Souza, G. A., Salte, T., Wiker, H. G., and Asjö, B. (2009). HIV induces both a downregulation of IRAK- 4 that impairs TLR signalling and an up-regulation of the antibiotic peptide dermcidin in monocytic cells. Scand. J. Immunol. 70, 264-276.

Quesniaux, V., Fremond, C., Jacobs, M., Parida, S., Nicolle, D., Yeremeev, V., et al. (2004). Toll-like receptor pathways in the immune responses to mycobacteria. Microbes Infect. 6, 946-959.

Randhawa, A. K., Shey, M. S., Keyser, A., Peixoto, B., Wells, R. D., de Kock, M., et al. (2011). Association of human TLR1 and TLR6 deficiency with altered immune responses to BCG vaccination in South African infants. PLoS Pathog. 7:e1002174. doi: 10.1371/journal.ppat.1002174

Ranjbar, S., Jasenosky, L. D., Chow, N., and Goldfeld, A. E. (2012). Regulation of Mycobacterium tuberculosis-dependent HIV-1 transcription reveals a new role for NFAT5 in the toll-like receptor pathway. PLoS Pathog. 8:e1002620. doi: 10.1371/journal.ppat.1002620

Shin, D. M., Yuk, J. M., Lee, H. M., Lee, S. H., Son, J. W., Harding,
C. V., et al. (2010). Mycobacterial lipoprotein activates autophagy via TLR2/1/CD14 and a functional vitamin D receptor signaling. Cell. Microbiol. 12, 1648-1665.

Singh, S. B., Davis, A. S., Taylor G. A., and Deretic, V. (2006). Human IRGM induces autophagy to eliminate intracellular mycobacteria. Science 313, 1438-1441.

Stenger, S., and Modiln, R. L. (2002) Control of Mycobacterium tuberculosis through mammalian Toll-like receptors. Curr. Opin. Immunol. 14 452-457.

Sundaramurthy, V., and Pieters, J. (2007). Interactions of pathogenic mycobacteria with host macrophages. Microbes Infect. 9, 1671-1679.

Texereau, J., Chiche, J. D., Taylor, W. Choukroun, G., Comba, B., and Mira, J. P. (2005). The importance of Toll-like receptor 2 polymorphisms in severe infections. Clin. Infect. Dis. 41, 408-415.

Werninghaus, K., Babiak, A., Groß2, O., Hölscher, C., Dietrich, H., Agger, E. M., et al. (2009). Adjuvanticity of a synthetic cord factor analogue for subunit Mycobacterium tuberculosis vaccination requires $\mathrm{FcR} \gamma-\mathrm{Syk}-$ Card9-dependent innate immune activation. J. Exp. Med. 206, 89-97.

WHO. (2010). "Multidrug and extensively drug-resistant TB (M/XDR-TB): 2010 global report on surveillance and response," in WHO Reports (Geneva, Switzerland: WHO).

Xu, Y., Jagnnath, C., Liu, X. D., Sharafkhaneh, A., Kolodziejska, K. E., and Eissa, N. T. (2007). Toll-like receptor 4 is a sensor for autophagy associated with innate immunity. Immunity 27, 135-144.

Yang, C. S., Shin, D. M., Kim, K. H. Lee, Z. W., Lee, C. H., Park, S. G., et al. (2009). NADPH oxidase 2 interaction with TLR2 is required for efficient innate immune responses to mycobacteria via cathelicidin expression. J. Immunol. 182, 3696-3705.

Yoshida, A., Inagawa, H., Kohchi, C., Nishizawa, T., and Soma, G. (2009). The role of toll-like receptor 2 in survival strategies of Mycobacterium tuberculosis in macrophage phagosomes. Anticancer Res. 29, 907-910.

Yuk, J. M., Shin, D. M., Lee, H. M., Yang, C. S., Jin, H. S., Kim, K. K., et al. (2009). Vitamin D3 induces autophagy in human monocytes/macrophages via cathelicidin. Cell Host Microbe 6, 231-243.

Yuk, J. M., Yoshimori, T., and Jo, E. K. (2012). Autophagy and bacterial infectious diseases. Exp. Mol. Med. 44, 99-108.

Zullo, A. J., and Lee, S. (2012). Old antibiotics target TB with a new trick. Cell Host Microbe 11, 419-420.

Zuñiga, J., Torres-Garcia, D., SantosMendoza, T., Rodriguez-Reyna, T. S., Granados, J., and Yunis, E. J. (2012). Cellular and humoral mechanisms involved in the control of tuberculosis. Clin. Dev. Immunol. 2012, 193923.

Conflict of Interest Statement: The authors declare that the research was conducted in the absence of any commercial or financial relationships that could be construed as a potential conflict of interest.

Received: 01 August 2012; accepted: 06 November 2012; published online: 23 November 2012.

Citation: Basu J, Shin D-M and Jo E-K (2012) Mycobacterial signaling through toll-like receptors. Front. Cell. Inf. Microbio. 2:145. doi: 10.3389/fcimb. 2012.00145

Copyright (C) 2012 Basu, Shin and Jo. This is an open-access article distributed under the terms of the Creative Commons Attribution License, which permits use, distribution and reproduction in other forums, provided the original authors and source are credited and subject to any copyright notices concerning any third-party graphics etc. 